Article

\title{
Multi-Objective Optimization for Reservoir Operation Considering Water Diversion and Power Generation Objectives
}

\author{
Xiaomei Sun ${ }^{\circledR}$, Jungang Luo * and Jiancang Xie \\ State Key Laboratory of Eco-Hydraulics in Northwest Arid Region, Xi'an University of Technology, Xi'an 710048, \\ China; xiaomeisun1020@foxmail.com (X.S.); jcxie@xaut.edu.cn (J.X.) \\ * Correspondence: jgluo@xaut.edu.cn; Tel.: +86-029-8231-2494
}

Received: 20 September 2018; Accepted: 26 October 2018; Published: 29 October 2018

check for updates

\begin{abstract}
Due to the uneven distribution of water resources in time and space, the problem of water shortage has become increasingly serious in some areas. To optimize use of water resources, it is urgent to establish multi-objective models and apply effective optimization algorithms to guide reservoir management. This study proposed a model of multi-objective optimization for reservoir operation (MORO) with the objectives of maximizing water diversion and power generation. The multi-objective evolutionary algorithm based on decomposition with adaptive weight vector adjustment (MOEA/D-AWA) was applied to solve the MORO problem. In addition, the performance of the MOEA/D-AWA was compared with two other algorithms based on the hyper-volume index. Huangjinxia reservoir, which is located in Shaanxi, China, was selected as the case study. The results show that: (1) the proposed model is effective and reasonable in theory; (2) the optimization results obtained by MOEA/D-AWA demonstrate this algorithm can be applied to the MORO problem, providing a set of evenly distributed non-dominated solutions; and (3) water diversion and power generation are indeed contradictory objectives. The MORO strategy can be used to efficiently utilize water resources, improve the comprehensive benefits of reservoirs, and provide decision support for actual reservoir operation.
\end{abstract}

Keywords: multi-objective optimization; reservoir operation; water diversion; power generation; MOEA/D-AWA

\section{Introduction}

With the progress of society, economic development, and the growth of the population, the problems of the water shortage and the imbalance of water supply and demand have become increasingly prominent. Human activity and land use can affect water resources. The effects can be explained by connectivity thinking: that is, human activities directly affect connectivity by land cover changes and different land use practices altering sediment delivery from hillslopes and floodplains to the channel system [1-5]. Water resource systems are very complicated, and the benefits of water resources should be maximized in terms of management and utilization of water resources. Thus, it is necessary to engage in management actions to fulfill consumer needs to achieve sustainable development and maximize benefits, especially in areas where the imbalance problem is serious [6]. Reservoirs are one of the most efficient key infrastructure components in water resources development and management [6,7]. Since water conservation projects often perform many tasks, such as flood control, power generation, meeting ecological needs, and providing water supply, etc., a single-objective operation based solely on water supply or power generation can no longer meet actual needs. Therefore, it has become increasingly obvious that the needs of multiple objectives 
should be met simultaneously to maximize the comprehensive benefits of water conservancy projects. Reservoir optimization operation requires establishing a model of reservoir optimization operation and then exploring the optimization algorithm of the model. Thus, this study studied the multi-objective optimization for reservoir operation (MORO) problem from these two perspectives.

Multi-objective reservoirs are often used to serve multiple demands for domestic, industrial, irrigation, environment, hydropower production, and flood control and so on to maximize economic benefits. Determining how to best meet these multiple needs is a complex problem because of the nonlinear storage-inflow relationship, conflicting objectives, dynamic properties, and nonlinear constraints, etc. [8]. During the past several decades, there have been many MORO studies. Most have mainly focused on meeting the bi-objectives and tri-objectives of reservoirs, such as power generation and water supply [9], flood control operation [10,11], power generation and ecology [12] and so on. At the same time, there have been significant advances in the development of optimization methods. The classical optimization methods for reservoir operation mainly include linear programming (LP) [13,14], non-linear programming (NLP) [15], dynamic programming (DP) [6,16,17], and quadratic programming [18]. These algorithms can solve small-scale optimization problems quickly. However, with the increase of the scale of the problems, the quality of the solutions is not sufficient using these algorithms.

With the emergence and rapid development of computational intelligence, evolutionary algorithms (EAs) have become popular for use in optimization of reservoir operation. EAs include artificial neural networks [19,20], the genetic algorithm [21], particle swarm optimization [22], ant colony optimization [23], honey bee mating optimization [24], and so on. In recent years, multi-objective evolutionary algorithms have been successfully applied to the MORO problem to obtain a set of Pareto optimal solutions in single run, which are best tradeoff solutions among different objectives. At present, multi-objective optimization evolutionary algorithms can be summarized into three categories: Pareto-dominance-based algorithms like non-dominated sorting genetic algorithm II (NSGA-II) [25] and strength Pareto evolutionary algorithm II (SPEA2) [26], indicator-based algorithms like IBEA [27], and decomposition-based algorithms like multi-objective evolutionary algorithm based on decomposition (MOEA/D) [10,28].

MOEA/D was originally proposed by Zhang and Li [28]. In MOEA/D, multi-objective optimization problems are decomposed into a number of scalar optimization sub-problems that are then optimized simultaneously. In addition, the diversity of the obtained solutions is guaranteed with the use of weight design methods and the use of neighbor information in the MOEA/D approach [29]. Due to its simplicity and high efficiency, this algorithm has been applied to many fields. However, MOEA/D also faces difficulties in addressing multi-objective optimization problems with complicated Pareto fronts. Some studies on the improvement of MOEA/D have also been carried out, such as modified MOEA/D [29], multi-objective evolutionary algorithm based on decomposition with opposition-based learning (MOEA/D-OBL) [30], self-adaptive multi-objective evolutionary algorithm based on decomposition (SaMOEA/D) [31], multi-objective evolutionary algorithm with decomposition and preference (MOEA/D-PWA) [32], MOEA/D-AWA [33] and others. MOEA/D-AWA introduces a new weight vector initialization method and an adaptive weight vector adjustment strategy. The weights are adjusted periodically to adaptively redistribute the weights of subproblems to obtain better uniformity of solutions [33].

Although these studies have yielded rich results for MORO problems and optimization algorithms, there are a few studies on MORO to balance the objectives of maximizing water diversion and maximizing power generation. Moreover, MOEA/D-AWA has not been applied to the MORO problem. Therefore, the goal of this study was to study the MORO problem and establish the model with the objectives of maximizing water diversion and maximizing power generation, and apply the MOEA/D-AWA algorithm to address and optimize the problem. Because NSGA-II is a very classical genetic algorithm, is one of the most popular multi-objective genetic algorithms at present, and it becomes the benchmark of performance of other multi-objective optimization algorithms [25,34], 
and MOEA/D-AWA is an improved algorithm for MOEA/D, the results obtained by MOEA/D-AWA were compared with the results of NSGA-II and MOEA/D, and algorithm performance was measured based on the Hyper-volume index [35-37]. The Huangjinxia reservoir, which is located in Shaanxi, China, was the focus of the case study. It's meaningful for improving the comprehensive benefits of water conservancy projects to verify whether the proposed model is successful and the introduced algorithm has potential to solve the MORO problem. Better models and appropriate algorithms can help ensure that water resources are used efficiently and reasonably, and can effectively guide decisions on actual reservoir operation.

The rest of this paper is organized as follows. Section 2 proposes and establishes the reservoir operation optimization model, and considers some constraints. Section 3 introduces the MOEA/D-AWA algorithm, and presents the basic idea and the procedure of application of MOEA/D-AWA for the MORO problem. Section 4 describes the Huangjinxia reservoir as the focus of the case study, presenting the parameters and data of the model and the algorithm. Section 5 compares the performance of three algorithms, and analyzes and discusses the rationality of the operation results. Section 6 concludes the paper.

\section{Reservoir Operation Optimization Model}

Generally, reservoirs can be used to irrigation, municipal and industrial water supply, hydropower generation, flood control, and other uses. Therefore, the management of a multi-objective reservoir is complex due to conflicts between these objectives [38]. Reservoir optimization operation can be used for formulating, analyzing, and solving operation optimization problems in water resources planning and management [39]. The MORO problem aims to maximize water resources benefits as much as possible by determining the optimal release for each reservoir over the entire operation period, while satisfying appropriate physical and operational constraints [40]. The objective functions and associated constraints of the MORO problem studied in the paper can be expressed as follows.

\subsection{Objective Functions}

In order to rationally and effectively use the water resources of a reservoir, a multi-objective optimization model is required that considers both water diversion and power generation objectives for reservoirs with water diversion and power generation tasks [9]. The monthly water levels are taken as the decision variables. Due to the fact that the water storage is reflected by the water level, the fluctuation of the water level represents the variation of the outflow [7]. The amount of water for water diversion and power generation is dynamically determined by the water level for each period, and also affects the water level of the reservoir in turn. Once the upstream water level is determined, the amount of available water can be determined through the water balance equation shown in Equation (3). Simultaneously, the downstream water level is connected with the outflow of the reservoir. In terms of the hydroelectric power station, the increase of the outflow will result in a higher downstream water level, thus leading to a lower hydraulic head. In contrast, as for the pump station, the increase of the outflow will result in a lower upstream water level, thus leading to a higher hydraulic head for water diversion. Thus, the two objective functions have obvious conflict. These two objective functions are expressed as follows:

1. Maximum water diversion:

$$
\operatorname{Max} f_{1}=\max \sum_{t}^{T} Q_{w d}(t) \Delta T(t),
$$

where $Q_{w d}(t)$ is the water diversion flow; $\Delta T(t)$ is the time step; $t$ is the index of operational periods; and $T$ is the number of operational periods in one year. 
2. Maximum power generation:

$$
\operatorname{Max} f_{2}=\max \sum_{t}^{T} K Q_{f d}(t) H(t) \Delta T(t)
$$

where $K$ is the power coefficient; $Q_{f d}(t)$ is the release flow passing through the turbines; $H(t)$ is the hydraulic head of a hydropower plant.

\subsection{Constraints}

The above objective functions of the model on reservoir optimization operation are subject to the following constraints:

(1) Water balance constraint:

$$
V(t+1)=V(t)+\left(I(t)-Q_{w d}(t)-Q_{f d}(t)-S(t)\right) * \Delta T(t), \forall t \in T,
$$

where $V(t+1)$ and $V(t)$ are the final and initial storage volumes during period; respectively; $I(t)$ is the inflow into the reservoir during each period $\Delta T(t)$; and $S(t)$ is the amount of the loss of the reservoir during each period $\Delta T(t)$.

(2) Storage of the reservoir constraint:

$$
V_{\min }(t) \leq V(t) \leq V_{\max }(t), \forall t \in T,
$$

where $V_{\min }(t)$ and $V_{\max }(t)$ are the minimum and maximum storage allowed in each period, respectively. The minimum storage $V_{\min }(t)$ is considered equal to the dead storage. $V_{\max }(t)$ is constrained by flood protection rules during the flood periods when the maximum storage $V_{\max }(t)$ is equivalent to the corresponding storage capacity of the flood-limited level of the reservoir. In the remaining periods, the maximum storage $V_{\max }(t)$ is equivalent to the utilizable capacity of the reservoir.

(3) Power output constraint:

$$
N_{\min } \leq N(t) \leq N_{\max }, \forall t \in T,
$$

where $N_{\min }$ and $N_{\max }$ are the minimum and maximum power output of the hydropower station, respectively.

(4) Release constraint:

$$
Q_{\min } \leq Q_{f d}(t) \leq Q_{\max }, \forall t \in T,
$$

where $Q_{\min }$ and $Q_{\max }$ are the minimum and maximum turbine capacity, respectively.

(5) Pumping station design flow constraint:

$$
Q_{w d}(t) \leq Q_{\text {design }}, \forall t \in T,
$$

where $Q_{\text {design }}$ is the pumping station design flow.

(6) Nonnegative constraints:

All values above are positive.

\section{Methodology}

With the above model and constraints, the appropriate optimization algorithm is applied to optimize the MORO problem. In this study, MOEA/D-AWA was adopted to the MORO problem with the proposed operation model, and the monthly water level values of the reservoir were selected as the decision variables. 


\subsection{MOEA/D-AWA}

MOEA/D-AWA was adopted to address the MORO problem to obtain a uniformly distributed optimal solution set on the Pareto front of the objectives. MOEA/D-AWA has the same framework as MOEA/D, but is enhanced with inclusion of a new weight vector initialization method and an adaptive weight vector adjustment (AWA) strategy. Here, the new weight vector initialization method is based on the geometric analysis of the Chebyshev decomposition approach, and the adaptive weight vector adjustment (AWA) strategy is to deal with the multi-objective optimization problems with complex Pareto fronts [33]. A more detailed description of MOEA/D-AWA is written in Qi et al. (2014) [33].

The simplified flow chart of the MOEA/D-AWA is shown in Figure 1. In the initialization stage, the data, parameters and studied problem are input. After the utilizing the simulated binary crossover (SBX) operator, polynomial mutation operator, adaptive weight adjustment, and other steps, the stopping condition is finally satisfied and the optimal solutions are obtained. Here, the generation number is termed as gen, the external population is termed as $E P$, the evolving population is termed as evol_pop, and the adaptive weight vector adjustment strategy is termed as wag.

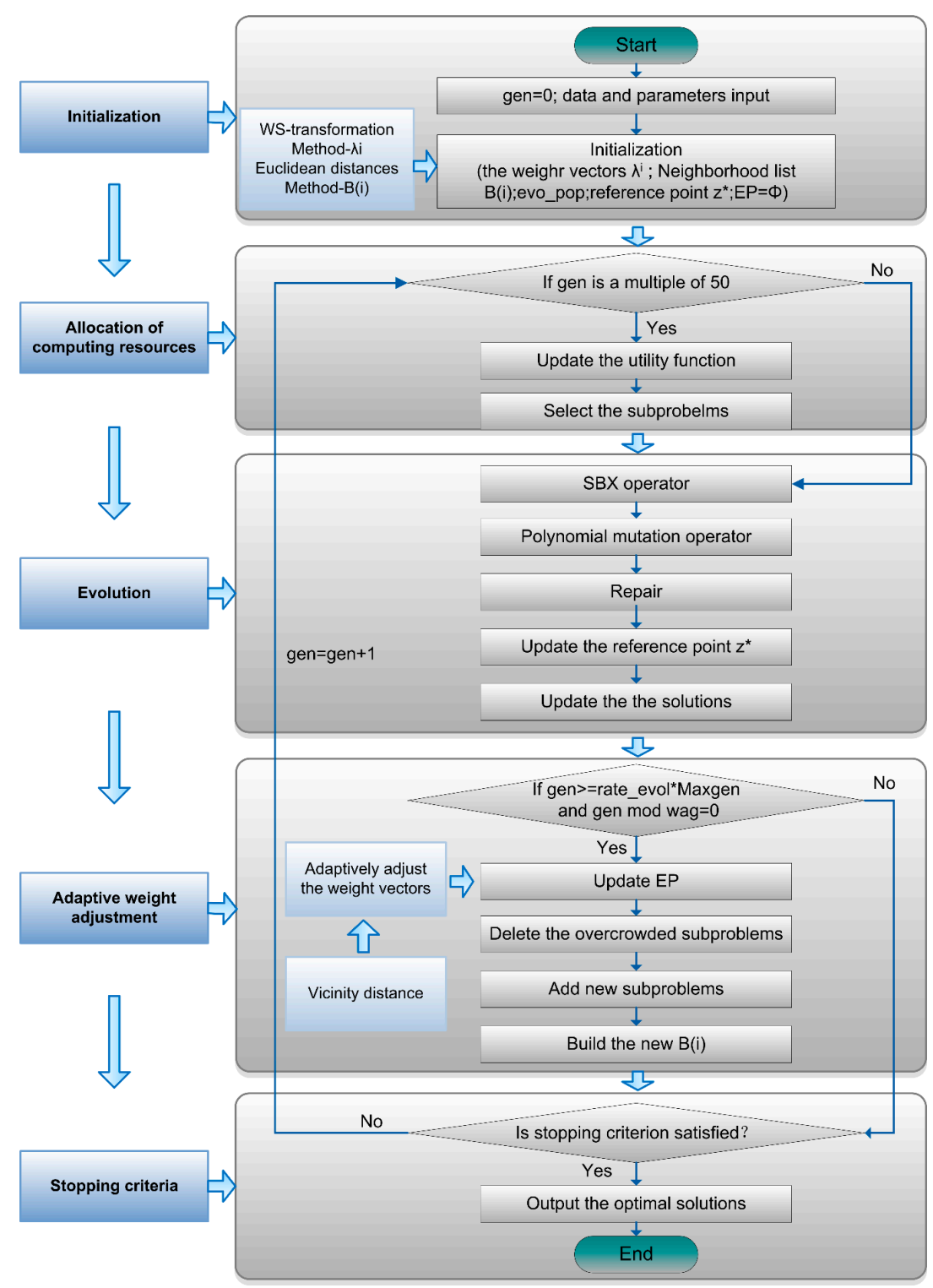

Figure 1. Simplified flow chart of the MOEA/D-AWA. 


\subsection{Application of MOEA/D-AWA for the MORO Problem}

Based on the basic ideas and basic steps of MOEA/D-AWA, the MOEA/D-AWA algorithm is applied to the MORO problem. The established functions and constraints of the MORO problem are added into the algorithm, and the established MORO model is used as the evaluation function. The population size is $N$, the maximum iteration time Maxgen is 800 , and the operation periods are 12 months. Water levels are taken as decision variables, and $\mathrm{N}$ individuals are randomly generated within the feasible ranges of water levels (the upper and lower limits of water levels). Under certain constraints, the quality of the solutions is evaluated according to the proposed objective functions. Through a series of steps, mainly including allocation of computing resources, evolution and adaptive weight adjustment, constantly update the solutions, so repeatedly, until the stopping criterion is satisfied. The steps of MOEA/D-AWA are shown in Algorithm 1.

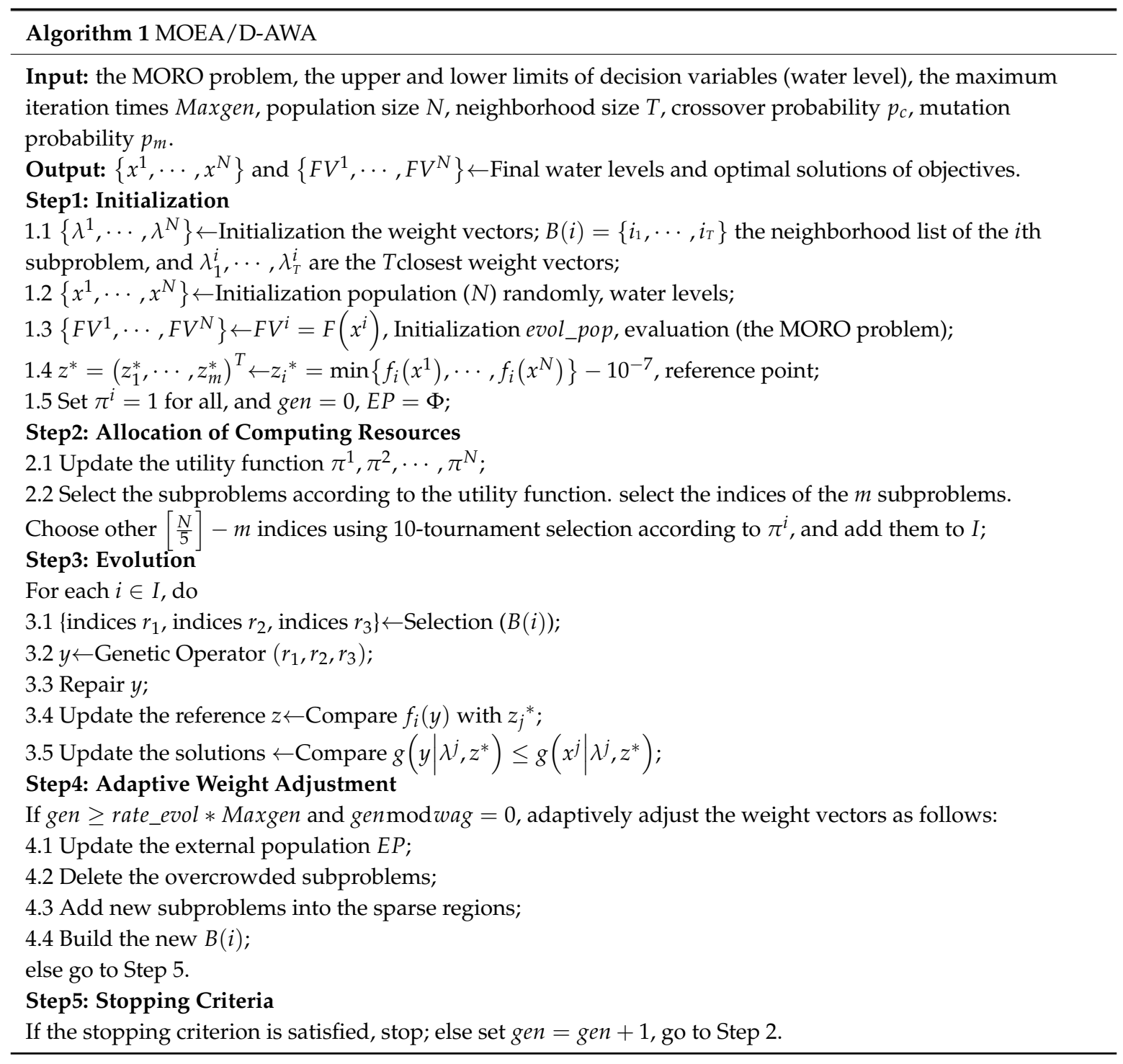

\section{Case Study}

\subsection{Study Area}

Shaanxi province in China has the two main drainage systems of the Yellow River and Yangtze River, bounded by the Qinling Mountains. As the largest tributary of the Yangtze River, the Hanjiang River originates from the Qinling Mountains in Shaanxi, China, with a total length of $1577 \mathrm{~km}$, 
covering $159,000 \mathrm{~km}^{2}$. The average annual precipitation of the river basin is 700-1100 mm, of which the upstream is about $800-1200 \mathrm{~mm}$. The runoff of the Hanjiang River is mainly supplied by precipitation, that mainly occurs in May-October. The Hanjiang river has abundant water resources, with 58.2 billion $\mathrm{m}^{3}$. The Weihe River, originating from the Gansu province in China and passing through the Shaanxi province in China, is the largest tributary of the Yellow River in China [41,42]. The total length of the Weihe River is $818 \mathrm{~km}$ and its drainage area is $135,000 \mathrm{~km}^{2}$, accounting for $17.9 \%$ of the total amount of the Yellow River basin. The main stream of the Weihe River is in Shaanxi, with a total length of $502.4 \mathrm{~km}$ and a drainage area of $67,108 \mathrm{~km}^{2}$. The average annual runoff of the Weihe River is 7.57 billion $\mathrm{m}^{3}$, and that of Shaanxi is 5.38 billion $\mathrm{m}^{3}$. The Weihe River plays an important role in Shaanxi, but its runoff has decreased significantly in recent decades [42].

The Hanjiang to Weihe River Water Diversion Project is located on the upper reach of the Hanjiang River in Shaanxi, China. The water diversion project is designed to divert water from the Hanjiang River with relatively abundant water resources into the Weihe River with severely deficient water resources. The main infrastructures of the water diversion project include two reservoirs (Huangjinxia reservoir and Sanhekou reservoir), two hydro plants, and two pumping stations.

Because the Huangjinxia reservoir with abundant inflow is the main source of water for Hanjiang to Weihe River Water Diversion Project, the case study focus is the Huangjinxia reservoir. The reservoir is located on the mainstream of the Hanjiang River, as shown in Figure 2. The Huangjinxia reservoir is a multi-objective reservoir, mainly taking on water supply, and also taking on power generation, flood control, and shipping. The reservoir supplies water to the Weihe River using the pumping station with a pumping capacity of $70 \mathrm{~m}^{3} / \mathrm{s}$. Moreover, the activity of water diversion is carried out by direct intake from the reservoir area by the pumping station. The reservoir will be dispatched out water from July to June next year, and the water level will be stored at a limited water level during the flood periods, otherwise, it will be stored at the normal water level. The variational water level will drop to the dead water level at the end of June next year. The basic parameters of the Huangjinxia reservoir, hydro plant, and pump station are presented in Table 1.
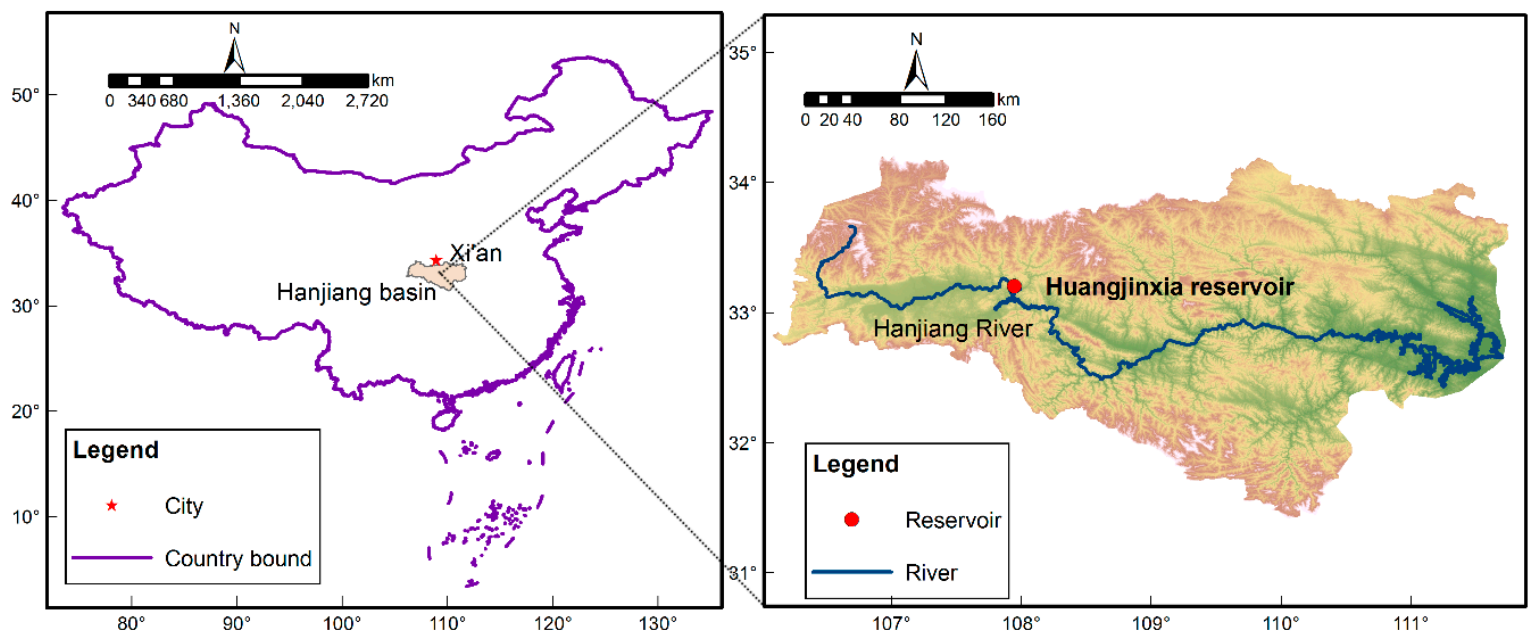

Figure 2. Location of the Huangjinxia reservoir. 
Table 1. Huangjinxia reservoir, hydro plant, and pump station characteristics.

\begin{tabular}{ccccc}
\hline Items & Units & Huangjinxia Reservoir & Hydro Plant & Pump Station \\
\hline Dead level & $\mathrm{m}$ & 440 & - & - \\
Normal pool level & $\mathrm{m}$ & 450 & - & - \\
Flood control level & $\mathrm{m}$ & 448 & - & - \\
Ecological demand & $\mathrm{m}^{3} / \mathrm{s}$ & 25 & - & - \\
Guaranteed output & $\mathrm{MW}$ & - & 85 & - \\
Installed capacity & $\mathrm{MW}$ & - & 135 & 126 \\
Maximum overflow & $\mathrm{m}^{3} / \mathrm{s}$ & - & 435.3 & 70 \\
\hline
\end{tabular}

\subsection{Model and Algorithm Application}

A monthly time scale was selected with a total time period of 12 months. The operation period was from July to June next year. The inflow data was selected with a probability of $75 \%$.

In the study, the MOEAD-AWA algorithm was introduced to solve the MORO problem. The results obtained by MOEAD-AWA algorithm were compared with two other multi-objective evolutionary algorithms, MOEA/D and NSGA-II. The optimization problem included 13 decision variables, which were the 13 water level values of the reservoir for the 12 time periods, including the starting water level and the ending water level.

MOEA/D-AWA, MOEA/D, and NSGA-II all used a simulated binary crossover operator and polynomial mutation. After many experiments and comparisons, the following parameters were adopted: the three compared algorithms had the same population size of 100, the neighborhood size was set as 20 for MOEA/D-AWA and MOEA/D; the maximum iteration time was 800 . The other parameter settings of the three algorithms are listed in Table 2 , where $\mathrm{n}$ is the number of variables.

Table 2. Parameters for MOEA/D-AWA, MOEA/D and NSGA-II algorithms.

\begin{tabular}{cccc}
\hline Parameters & MOEA/D-AWA & MOEA/D & NSGA-II \\
\hline Crossover probability $p_{c}$ & 0.9 & 0.9 & 0.9 \\
Distribution index for crossover & 20 & 20 & 20 \\
Mutation probability $p_{m}$ & $1 / \mathrm{n}$ & $1 / \mathrm{n}$ & $1 / \mathrm{n}$ \\
Distribution index for mutation & 20 & 20 & 20 \\
\hline
\end{tabular}

The single-objective problem is to find an optimal and unique point in a space of decision variables, while a multi-objective optimization problem is not likely to have a unique solution that optimizes all the objectives, but instead has a set of optimal solutions. The basic parameters of the reservoir, hydropower station and pumping station and monthly inflow data are input, and this study proposes a model with two objectives, including maximizing water diversion and maximizing power generation, and various constraints are used as boundary conditions. MOEA/D-AWA is adopted to obtain optimal solutions. The algorithm parameters are set, and the optimal results are finally obtained until the stop condition is reached. The results of MOEA/D-AWA are compared with the results of NSGA-II and MOEA/D. The results include the Pareto fronts and the Hyper-volume curves of the three algorithms, the variable values, the performance evaluation results and the comparison results. Figure 3 shows three basic steps for the application of the mathematical optimization model and the introduced MOEA/D-AWA algorithm. 


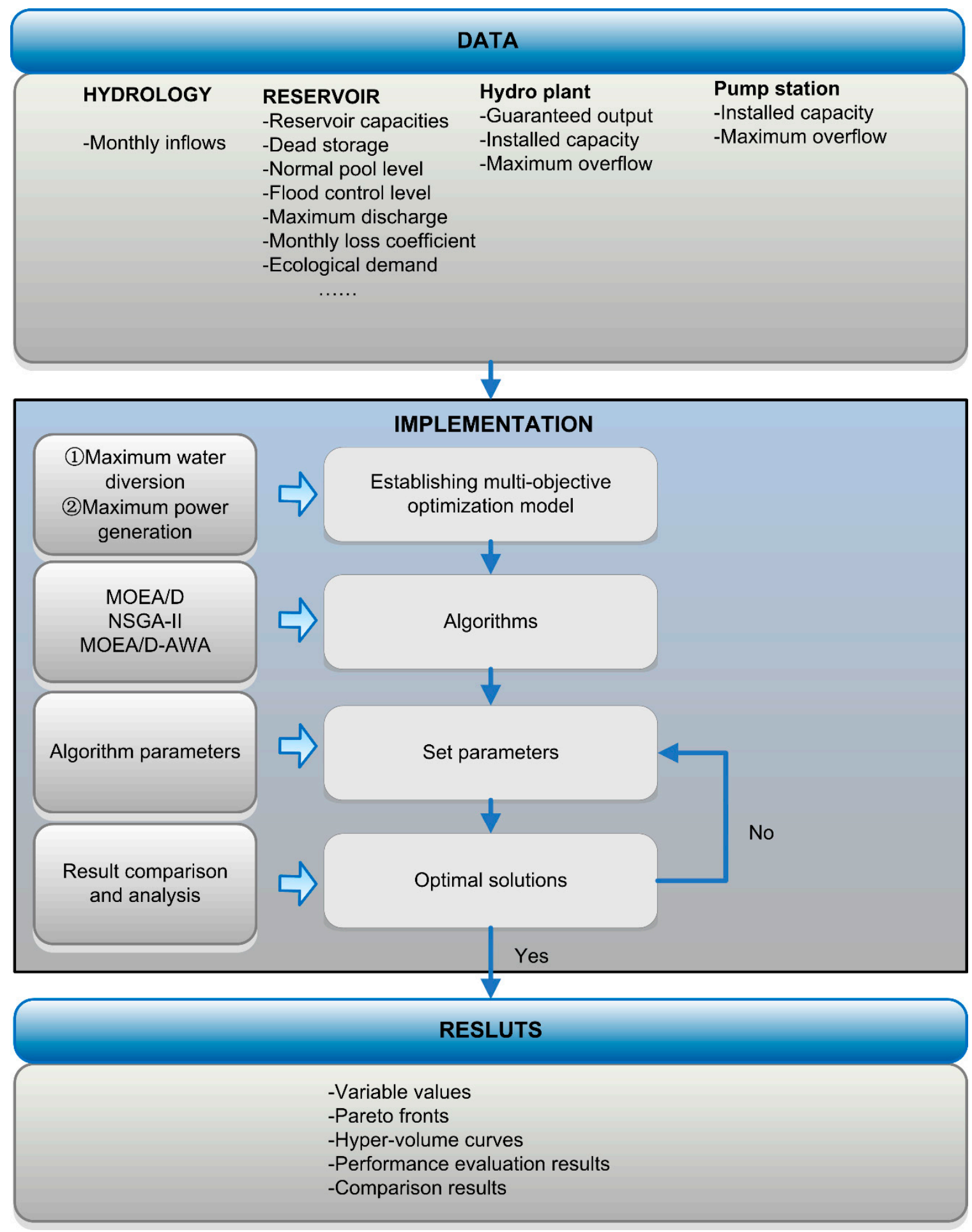

Figure 3. Model and algorithm application.

\section{Results}

The effectiveness of the proposed model and the feasibility of the introduced MOEA/D-AWA algorithm could be verified, by comparing the performance of different algorithms and analyzing the rationality of the operation results. 


\subsection{Performance of Different Algorithms}

1. Analysis of the Pareto distribution of different algorithms

Figure 4 showed the Pareto fronts of MOEA/D, NSGA-II, and MOEA/D-AWA algorithms for the MORO problem after 800 iterations. Overall, the trends of the three algorithms were roughly similar. However, as can be seen in Figure 4a, the Pareto front obtained by MOEA/D was discontinuously distributed. The non-dominated solutions obtained by MOEA/D were mainly concentrated at the bottom, but were discontinuously distributed at the top. As shown in Figures $4 \mathrm{~b}$ and $4 \mathrm{c}$, NSGA-II and MOEA/D-AWA performed significantly better than MOEA/D in terms of uniformity, and even the Pareto front distribution of MOEA/D-AWA was slightly more uniform than NSGA-II, due to slightly less uniformity at the top and bottom of NSGA-II's Pareto front compared to that of MOEA/D-AWA.

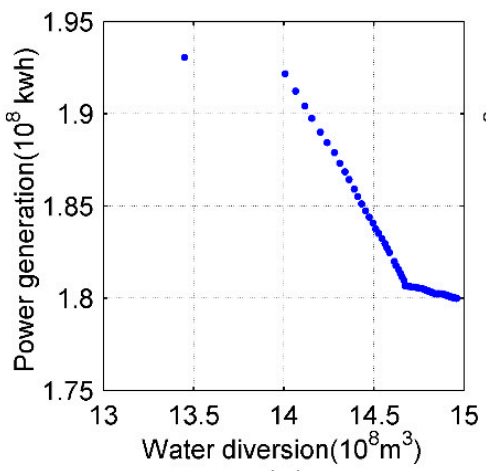

(a)

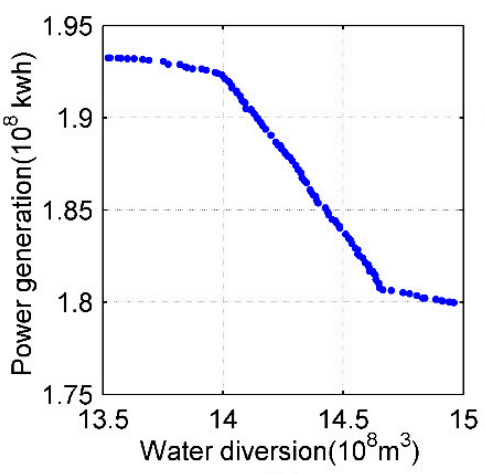

(b)

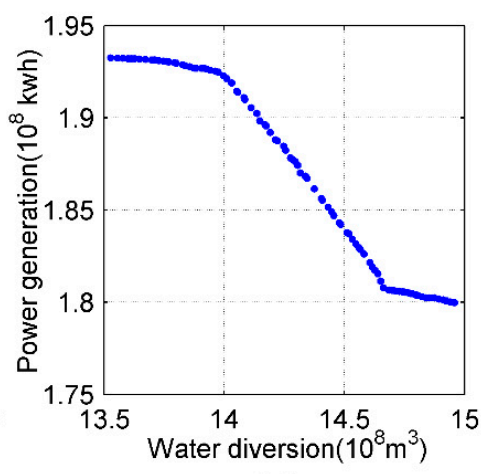

(c)

Figure 4. Pareto fronts of different algorithms for the proposed MORO problem: (a) Pareto front of MOEA/D; (b) Pareto front of NSGA-II; (c) Pareto front of MOEA/D-AWA.

\section{Analysis of convergence speed of different algorithms}

The hyper-volume (HV) is a comprehensive index to measure the quality of multi-objective optimization algorithms. Especially when the ideal Pareto front is unknown, the HV can objectively evaluate the convergence, breadth, and uniformity of the Pareto front $[35,36]$. For the minimization problem of two-objective optimization, the larger the value of the $\mathrm{HV}$, the better the convergence, breadth, and uniformity of the Pareto front. Additionally, a high HV value means that the non-dominated solution set is a good approximation of the unknown Pareto front [37].

As shown in Figure 5, with increased number of iterations, the convergence curves of $\mathrm{HV}$ of three algorithms became more stable. However, there were differences in the convergence speed and HV values of the three algorithms. The curve of MOEA/D started to stabilize after about 150 iterations, that of MOEA/D-AWA gradually stabilized after about 250 iterations, and the convergence rate for NSGA-II was slower than that of the other two. Before 600 iterations, the stability of the distribution of the non-dominated solution set of NSGA-II was relatively poor, and it gradually stabilized after 600 generations. After 600 or so iterations, the corresponding HV values of the three algorithms no longer increased with the increased number of iterations, and the distribution of the non-dominated solution sets was stable. The HV values are a measure of the quality of the non-dominated solution sets of algorithms. As shown in Figure 5, after 600 iterations, the HV value of MOEA/D-AWA was slightly higher than the HV values of MOEA/D and NSGA-II, which indicated that the distribution of the non-dominated solutions of MOEA/D-AWA was slightly more similar to the ideal Pareto front.

In general, although the convergence speed of MOEA/D was faster than that of the two other algorithms, the non-dominated solution distribution of MOEA/D was not uniform. By the above comparison and analysis, the convergence speed of MOEA/D-AWA was relatively fast, and the distribution of the non-dominated solution set of MOEA/D-AWA algorithm was uniform and closer to 
the theoretical Pareto front. Thus, the MOEA/D-AWA algorithm can be applied to the MORO problem and can achieve better results.

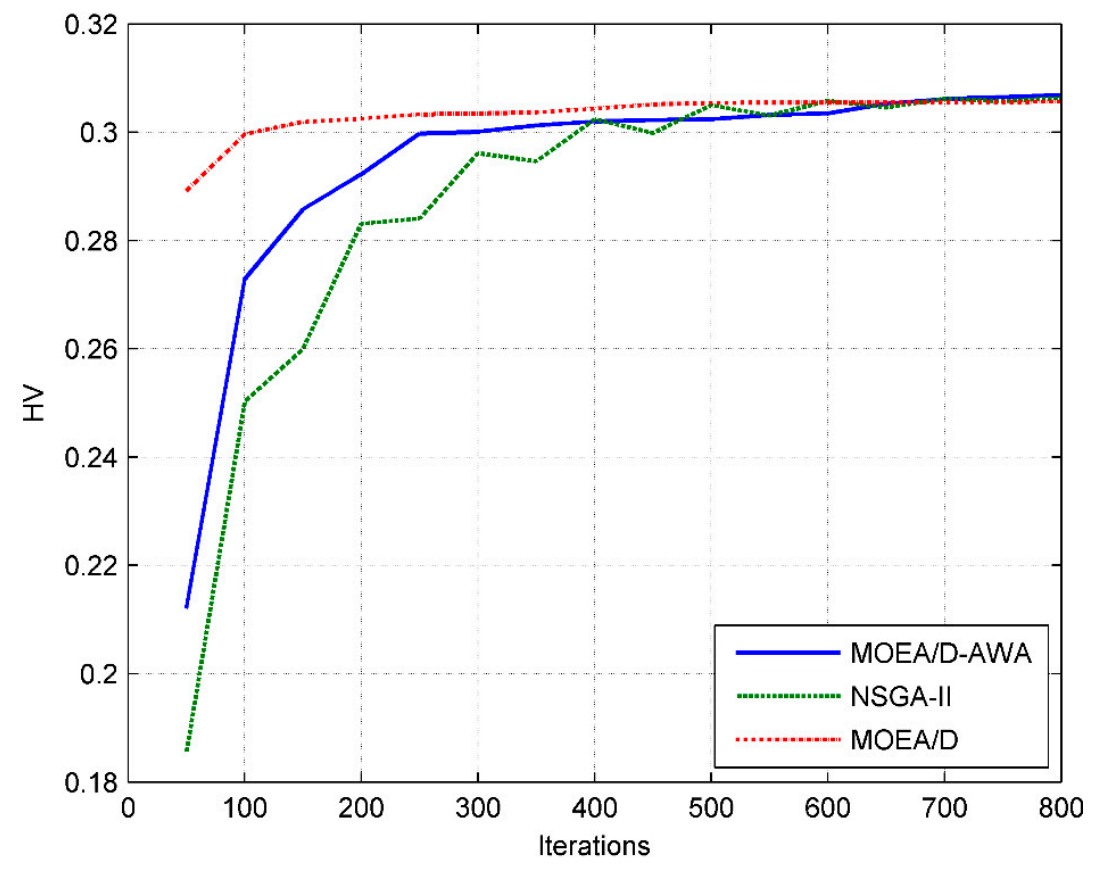

Figure 5. Hyper-volume index convergence curves of different algorithms.

\subsection{Rationality of the Operation Results}

The MOEA/D-AWA algorithm was used to optimize the MORO problem, providing a non-dominated solution set with uniform distribution. The solution set can provide decision support to coordinate the contradictory needs of the two objectives. Figure 6 shows the opposing objectives of water diversion and power generation: as water diversion increased, power generation decreased, and vice versa.

As shown in Figure 6, all the points on the Pareto front were optimal solutions. Selection among these solutions depends on the actual needs of reservoir operation and the choice of the decision makers. It is even possible to add another step in the process to weigh the optimal solutions. As evident in Figure 6, the AB and CD segments changed slowly, and the solutions corresponding to the four endpoints were obtained, namely solution A, solution B, solution C, and solution D. Solution A and solution $\mathrm{D}$ correspond to the maximum power generation and the maximum water diversion. As can be seen from Table 3, the water diversion from solution A to solution B underwent a large change, increasing by $3.275 \%$, while the power generation decreased only by $0.414 \%$. The water diversion from solution $C$ to solution $D$ underwent a very large change, with an increase of $1.866 \%$, and power generation decreased only by $0.387 \%$. To assess the comprehensive benefits of the reservoir, the economic benefits of the four solutions were calculated based on the water price of the water source of the Hanjiang to Weihe River Water Diversion Project, at 2 yuan $/ \mathrm{m}^{3}$ [9], and the water price of the hydropower station, at 0.3 yuan/kWh [43]. From solution A to solution B, the average annual economic benefits of the reservoir increased by $3.198 \%$; from solution $C$ to solution $D$, the average annual economic benefits of the reservoir increased by $1.825 \%$. Although all solutions on the Pareto front were optimal solutions, in terms of the AB and CD gentle segments, solution $B$ was better than solution $\mathrm{A}$ and other solutions in the $\mathrm{AB}$ segments, and solution $\mathrm{C}$ was better than solution $\mathrm{D}$ and other solutions in the CD segments in the actual reservoir operation, with greater comprehensive benefits of the reservoir. 


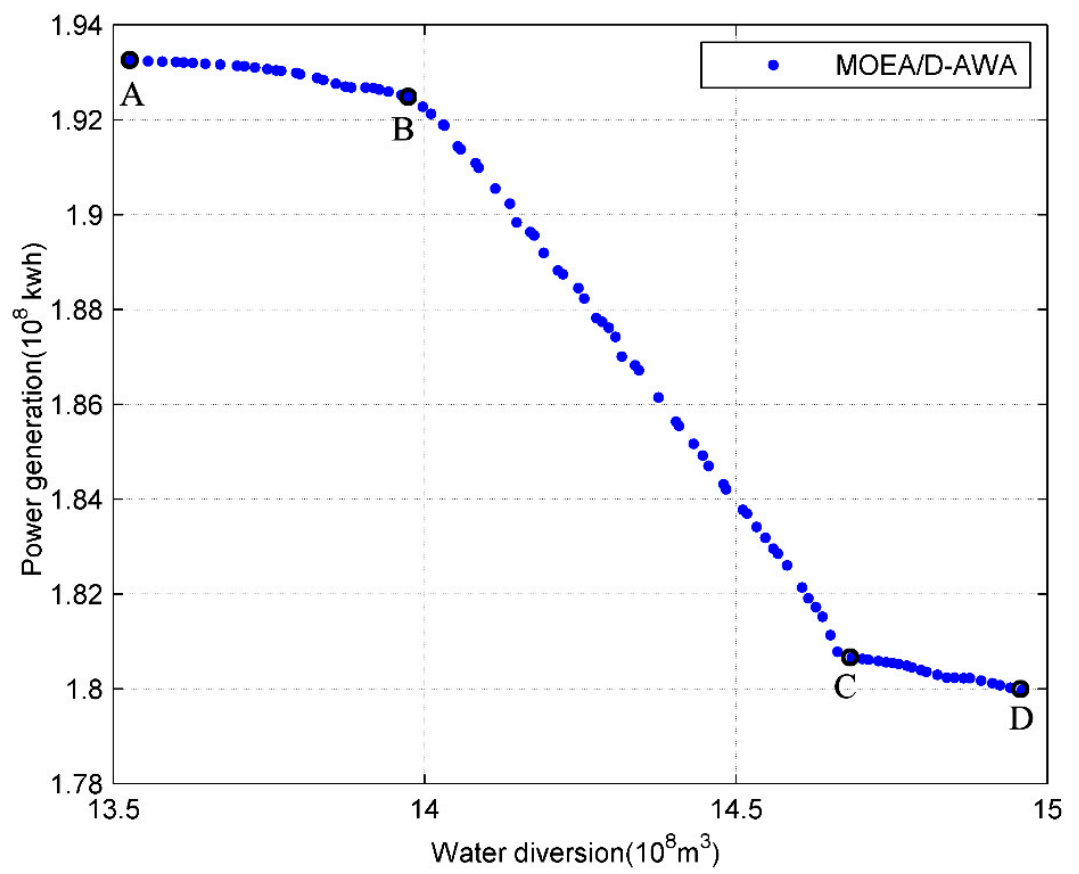

Figure 6. Pareto front of MOEA/D-AWA.

Table 3. Comprehensive benefit analysis of solutions on segments $A B$ and CD.

\begin{tabular}{ccccc}
\hline Items & A & B & C & D \\
\hline Water diversion $\left(10^{8} \mathrm{~m}^{3}\right.$ /year $)$ & 13.527 & 13.970 & 14.686 & 14.960 \\
Power generation $\left(10^{8} \mathrm{kWh} /\right.$ year $)$ & 1.933 & 1.925 & 1.807 & 1.800 \\
Economic benefit of reservoir $\left(10^{8}\right.$ yuan/year $)$ & 27.634 & 28.518 & 29.914 & 30.460 \\
Water diversion variation percentage $(\mathrm{A} \rightarrow \mathrm{B})$ & & $+3.275 \%$ & & $+1.866 \%$ \\
Power generation variation percentage $(\mathrm{A} \rightarrow \mathrm{B})$ & & $-0.414 \%$ & & $-0.387 \%$ \\
Economic benefit of reservoir variation & & $+3.198 \%$ & & $+1.825 \%$ \\
percentage $(\mathrm{A} \rightarrow \mathrm{B})$ & & & & \\
\hline
\end{tabular}

\section{Conclusions and Discussion}

Efficient reservoir operation can make full use of storage capacity of the reservoir and improve the comprehensive benefits of the reservoir. In this study, a model of the MORO problem was proposed to maximize water diversion and power generation objectives, with consideration of various constraints. The MOEA/D-AWA algorithm was applied to solve and optimize the MORO problem and compared with two other algorithms. This model was applied to the Huangjinxia reservoir in Shaanxi, China. The following conclusions can be drawn:

(1) The proposed reservoir operation model is effective and reasonable in theory, and can be used to improve the comprehensive benefits of the reservoir.

(2) The optimization results by obtained by MOEA/D-AWA, compared with MOEA/D and NSGA-II, indicate that MOEA/D-AWA can be applied to the MORO problem, providing a set of non-dominated solutions that quickly reach convergence and are evenly distributed.

(3) The use of the established model and MOEA/D-AWA shows that the two objectives of maximizing water diversion and maximizing power generation are in conflict. As water diversion increases, power generation decreases, and vice versa.

The effectiveness of the proposed model and the introduced algorithm is verified successfully. MOEA/D-AWA has the ability to solve the MORO problem. The results of this work provide a reference for MORO problems, can improve the comprehensive benefits of reservoirs and the utilization efficiency of water resources, thus alleviate the imbalance of water resources in time and space. This is 
a study for reservoirs with water diversion and power generation tasks. In future work, it will also be possible to combine with other objectives of reservoirs to build models and involve the multi-objective operation of cascade reservoirs. According to the Sustainable Development Goals (OSS/SDGs) of the UN 2030 agenda, the operation model can be continuously supplemented and improved. The decision support system for reservoir operation can be constantly integrated, and can even include spatial planning tools.

Author Contributions: Conceptualization, X.S. and J.L.; Data curation, J.L. and J.X.; Formal analysis, X.S. and J.L.; Funding acquisition, J.L. and J.X.; Investigation, X.S.; Methodology, X.S. and J.L.; Resources, J.L.; Software, X.S.; Supervision, J.L. and J.X.; Validation, X.S. and J.L.; Visualization, X.S.; Writing-original draft, X.S.; Writing-review and editing, X.S. and J.L.

Funding: This work was supported by the National Key R\&D Program of China under Grant No. 2016YFC0401409, and the National Natural Science Foundation of China under Grant Nos. 51679186 and 51679188.

Conflicts of Interest: The authors declare no conflict of interest.

\section{References}

1. Poeppl, R.E.; Keesstra, S.D.; Maroulis, J. A conceptual connectivity framework for understanding geomorphic change in human-impacted fluvial systems. Geomorphology 2017, 277, 237-250. [CrossRef]

2. Keesstra, S.D.; Kondrlova, E.; Czajka, A.; Seeger, M.; Maroulis, J. Assessing riparian zone impacts on water and sediment movement: A new approach. Neth. J. Geosci. 2012, 91, 245-255. [CrossRef]

3. Masselink, R.; Temme, A.J.A.M.; Giménez, R.; Casalí, J.; Keesstra, S.D. Assessing hillslope-channel connectivity in an agricultural catchment using rare-earth oxide tracers and random forests models. Cuad. Investig. Geogr. 2017, 43, 17-39. [CrossRef]

4. Keesstra, S.D.; Temme, A.J.A.M.; Schoorl, J.M.; Visser, S.M. Evaluating the hydrological component of the new catchment-scale sediment delivery model LAPSUS-D. Geomorphology 2014, 212, 97-107. [CrossRef]

5. Masselink, R.J.; Heckmann, T.; Temme, A.J.; Anders, N.S.; Gooren, H.P.; Keesstra, S.D. A network theory approach for a better understanding of overland flow connectivity. Hydrol. Process. 2017, 31, 207-220. [CrossRef]

6. Bozorg-Haddad, O.; Moravej, M.; Loaiciga, H.A. Application of the Water Cycle Algorithm to the Optimal Operation of Reservoir Systems. J. Irrig. Drain. Eng.-ASCE 2015, 141. [CrossRef]

7. Ming, B.; Chang, J.X.; Huang, Q.; Wang, Y.M.; Huang, S.Z. Optimal Operation of Multi-Reservoir System Based-On Cuckoo Search Algorithm. Water Resour. Manag. 2015, 29, 5671-5687. [CrossRef]

8. Al-Jawad, J.Y.; Tanyimboh, T.T. Reservoir operation using a robust evolutionary optimization algorithm. J. Environ. Manag. 2017, 197, 275-286. [CrossRef] [PubMed]

9. Ming, B.; Liu, P.; Chang, J.; Wang, Y.; Huang, Q. Deriving Operating Rules of Pumped Water Storage Using Multiobjective Optimization: Case Study of the Han to Wei Interbasin Water Transfer Project, China. J. Water Res. Plan. Man. 2017, 143, 05017012. [CrossRef]

10. Luo, J.G.; Sun, X.M.; Qi, Y.T.; Xie, J.C. Approximating the irregularly shaped Pareto front of multi-objective reservoir flood control operation problem. Appl. Math. Model. 2018, 54, 502-516. [CrossRef]

11. Luo, J.; Chen, C.; Xie, J. Multi-objective Immune Algorithm with Preference-Based Selection for Reservoir Flood Control Operation. Water Resour. Manag. 2014, 29, 1447-1466. [CrossRef]

12. Zhang, J.W.; Wang, X.; Liu, P.; Lei, X.H.; Li, Z.J.; Gong, W.; Duan, Q.Y.; Wang, H. Assessing the weighted multi-objective adaptive surrogate model optimization to derive large-scale reservoir operating rules with sensitivity analysis. J. Hydrol. 2017, 544, 613-627. [CrossRef]

13. Jabr, R.A.; Coonick, A.H.; Cory, B.J. A Homogeneous Linear Programming Algorithm for the Security Constrained Economic Dispatch Problem. IEEE Trans. Power Syst. 2000, 15, 930-936. [CrossRef]

14. Yoo, J.H. Maximization of hydropower generation through the application of a linear programming model. J. Hydrol. 2009, 376, 182-187. [CrossRef]

15. Basu, M. An interactive fuzzy satisfying method based on evolutionary programming technique for multiobjective short-term hydrothermal scheduling. Electr. Power Syst. Res. 2004, 69, 277-285. [CrossRef]

16. Travers, D.L.; Kaye, R.J. Dynamic dispatch by constructive dynamic programming. IEEE Trans. Power Syst. 1998, 13, 72-78. [CrossRef] 
17. Li, X.; Wei, J.H.; Li, T.J.; Wang, G.Q.; Yeh, W.W.G. A parallel dynamic programming algorithm for multi-reservoir system optimization. Adv. Water Resour. 2014, 67, 1-15. [CrossRef]

18. Papageorgiou, L.G.; Fraga, E.S. A mixed integer quadratic programming formulation for the economic dispatch of generators with prohibited operating zones. Electr. Power Syst. Res. 2007, 77, 1292-1296. [CrossRef]

19. Chaves, P.; Chang, F.J. Intelligent reservoir operation system based on evolving artificial neural networks. Adv. Water Resour. 2008, 31, 926-936. [CrossRef]

20. Chen, Y.H.; Chang, F.J. Evolutionary artificial neural networks for hydrological systems forecasting. J. Hydrol. 2009, 367, 125-137. [CrossRef]

21. Chang, L.C.; Chang, F.J.; Wang, K.W.; Dai, S.Y. Constrained genetic algorithms for optimizing multi-use reservoir operation. J. Hydrol. 2010, 390, 66-74. [CrossRef]

22. Marković, I.; Jurić-Kavelj, S.; Petrović, I. Partial mutual information based input variable selection for supervised learning approaches to voice activity detection. Appl. Soft Comput. 2013, 13, 4383-4391. [CrossRef]

23. Jalali, M.R.; Afshar, A.; Mariño, M.A. Multi-reservoir operation and adaptive pheromone re-initiated ant colony optimization algorithm. Int. J. Civ. Eng. 2007, 5, 284-301.

24. Bozorg Haddad, O.; Afshar, A.; Mariño, M.A. Multireservoiroptimisation in discrete and continuous domains. Proc. Inst. Civ. Eng. Water Manag. 2011, 164, 57-72. [CrossRef]

25. Deb, K.; Pratap, A.; Agarwal, S.; Meyarivan, T. A fast and elitist multiobjective genetic algorithm: NSGA-II. IEEE Trans. Evol. Comput. 2002, 6, 182-197. [CrossRef]

26. Lee, J.S.; Choi, L.C.; Park, S.C. Multi-Objective Genetic Algorithms, NSGA-II and SPEA2, for Document Clustering. Commun. Comput. Inf. Sci. 2011, 257, 219-227.

27. Chouikha, I.; Bree, A.; Moulin-Schouleur, M.; Gilot, P.; Germon, P. Differential expression of iutA and ibeA in the early stages of infection by extra-intestinal pathogenic E. coli. Microbes Infect. 2008, 10, 432-438. [CrossRef] [PubMed]

28. Zhang, Q.; Li, H. MOEA/D: A Multiobjective Evolutionary Algorithm Based on Decomposition. IEEE Trans. Evol. Comput. 2007, 11, 712-731. [CrossRef]

29. Zhang, J.; Tang, Q.; Li, P.; Deng, D.; Chen, Y. A modified MOEA/D approach to the solution of multi-objective optimal power flow problem. Appl. Soft Comput. 2016, 47, 494-514. [CrossRef]

30. Ma, X.L.; Liu, F.; Qi, Y.T.; Gong, M.G.; Yin, M.L.; Li, L.L.; Jiao, L.C.; Wu, J.S. MOEA/D with opposition-based learning for multiobjective optimization problem. Neurocomputing 2014, 146, 48-64. [CrossRef]

31. Qi, Y.T.; Bao, L.; Ma, X.L.; Miao, Q.G.; Li, X.D. Self-adaptive multi-objective evolutionary algorithm based on decomposition for large-scale problems: A case study on reservoir flood control operation. Inform. Sci. 2016, 367, 529-549. [CrossRef]

32. Qi, Y.T.; Yu, J.S.; Li, X.D.; Wei, Y.X.; Miao, Q.G. Reservoir flood control operation using multi-objective evolutionary algorithm with decomposition and preferences. Appl. Soft Comput. 2017, 50, 21-33. [CrossRef]

33. Qi, Y.T.; Ma, X.L.; Liu, F.; Jiao, L.C.; Sun, J.Y.; Wu, J.S. MOEA/D with Adaptive Weight Adjustment. Evol. Comput. 2014, 22, 231-264. [CrossRef] [PubMed]

34. Dugardin, F.; Yalaoui, F.; Amodeo, L. New multi-objective method to solve reentrant hybrid flow shop scheduling problem. Eur. J. Oper. Res. 2010, 203, 22-31. [CrossRef]

35. Knowles, J.; Corne, D. Properties of an adaptive archiving algorithm for storing nondominated vectors. IEEE Trans. Evol. Comput. 2003, 7, 100-116. [CrossRef]

36. Zitzler, E.; Thiele, L. Multiobjective evolutionary algorithms: A comparative case study and the strength Pareto approach. IEEE Trans. Evol. Comput. 1999, 3, 257-271. [CrossRef]

37. Yang, G.; Guo, S.L.; Liu, P.; Li, L.; Liu, Z. PA-DDS algorithm for multi-objective reservoir operation. J. Hydraul. Eng. 2016, 47, 789-797. [CrossRef]

38. Lin, N.M.; Rutten, M. Optimal Operation of a Network of Multi-Purpose Reservoir: A Review. Procedia Eng. 2016, 154, 1376-1384. [CrossRef]

39. Zhang, Z.B.; Jiang, Y.Z.; Zhang, S.H.; Geng, S.M.; Wang, H.; Sang, G.Q. An adaptive particle swarm optimization algorithm for reservoir operation optimization. Appl. Soft Comput. 2014, 18, 167-177. [CrossRef]

40. Papageorgiou, M. Optimal Multireservoir Network Control by the Discrete Maximum Principle. Water Resour. Res. 1985, 21, 1824-1830. [CrossRef] 
41. Chang, J.X.; Wang, Y.M.; Istanbulluoglu, E.; Bai, T.; Huang, Q.; Yang, D.W.; Huang, S.Z. Impact of climate change and human activities on runoff in the Weihe River Basin, China. Quat. Int. 2015, 380, 169-179. [CrossRef]

42. Du, J.; Shi, C.X. Effects of climatic factors and human activities on runoff of the Weihe River in recent decades. Quat. Int. 2012, 282, 58-65. [CrossRef]

43. Zhang, W. Based on the Theory of Two-part Water Price Analysis of Water Price in the water receiving area of Hanjiang to Weihe River Water Diversion Project. Ground Water 2013, 35, 101-117. 